\title{
Structural Aspects of Organic Superconductors
}

\section{Schweitzer \\ 3. Physikalisches Institut der Universität Stuttgart, Pfaffenwaldring 57, \\ D-7000 Stuttgart 80, Fed. Rep. of Germany}

Abstract. Some physical properties of the organic superconductors with the highest transition temperatures are presented. In addition the influence of small structural changes on the superconducting transition temperature is discussed. Finally bulk superconductivity in polycrystalline pressed pellets of organic metals is reported.

\section{Introduction}

In 1980 superconductivity in an organic metal was observed for the first time [1]. Today about 35 different organic metals are known which become superconducting under pressure or ambient pressure. The organic superconductors with the highest transition temperatures are all radical salts of the donor bis(ethylenedithiolo) tetrathiofulvalene (BEDT-TTF), which are at ambient pressure $(\text { BEDT - TTF })_{2} \mathrm{Cu}(\mathrm{NCS})_{2}\left(\mathrm{~T}_{\mathrm{c}}=10.4 \mathrm{~K}\right)[2]$ and $\alpha_{\mathrm{t}}-(\mathrm{BEDT}-\mathrm{TTF})_{2} \mathrm{I}_{3}$ $\left(T_{C}=8 \mathrm{~K}\right)[3]$ and under isotropic pressure $\beta_{H^{-}}(B E D T-T T F)_{2} I_{3}$ $\left(0.5 \mathrm{kbar}, \mathrm{T}_{\mathrm{c}}=7.5 \mathrm{~K}\right)[4.5]$.

In the following some of the structural and physical properties of single crystals of the above-mentioned organic metals are discussed briefly. In the second part a few new results and observations such as bulk superconductivity in polycrystalline pressed samples of the same materials are presented. The latter observation is remarkable since it shows that organic superconductors can be used in principle also for applications such as the preparation of electronic devices (SQUIDS) and might even be suitable for the preparation of superconducting cables. In addition it indicates that the observation of superconductivity in organic polymers should be possible as well.

\section{Properties of Single Crystals}

$(\mathrm{BEDT}-\mathrm{TTF})_{2} \mathrm{Cu}(\mathrm{NCS})_{2}$ is a typical quasi two-dimensional organic metal. The two-dimensional behaviour is due to conducting sheets of dimerised BEDT-TTF molecules which are separated 
by non-conducting anion layers. In the BEDT-TTF sheets the dimers are nearly orthogonally arranged with respect to each other [2] and the dimers do not form the usual face to face piling along the stacking axis. The latter property increases the twodimensional character of the molecular network. An important property of $(\mathrm{BEDT}-\mathrm{TTF})_{2} \mathrm{Cu}(\mathrm{NCS})_{2}$ is that the crystals show - compared to other organic superconductors - a relatively sharp transition into the superconducting state. This is observed not only in resistivity $[2,6]$ but also in the ac-susceptibility measurement [6] and in the Meissner effect [2,7]. The two latter methods have shown that superconductivity in $(\mathrm{BEDT}-\mathrm{TTF})_{2} \mathrm{Cu}(\mathrm{NCS})_{2}$ is a nearly $100 \%$ bulk effect. Measurements of the upper critical fields have demonstrated the typical quasi two-dimensional character of the crystals $[8,9]$. It could be shown that at temperatures below $4 \mathrm{~K}$ the superconducting properties can be described best in the picture of layered two-dimensional superconductors as first reported by Klemm et al. [10]. This picture of layered two-dimensional superconductors is valid for the high $T_{c}$ copper oxide superconductors as well and demonstrates the similarities of the structural principles of both classes of materials. These similarities will be discussed in the following in more detail for the example of the organic superconductor $\alpha_{t}-$ (BEDTTTF) ${ }_{2} \mathrm{I}_{3}$.

Crystals of $\alpha_{t}-(B E D T-T T F)_{2} I_{3}$ are quasi two-dimensional organic metals as well which have a stable superconducting state at $8 \mathrm{~K}$ and ambient pressure [3]. The crystals can be prepared by annealing crystals of $\alpha-(\text { BEDT }-T T F)_{2} I_{3}$ above $70^{\circ} \mathrm{C}$ for several days, resulting in a structural phase transition. The structure of $\alpha_{t}-(B E D T-T T F)_{2} I_{3}$ is very similar to that of crystals of $\beta-(B E D T-T T F)_{2} I_{3}$ as was shown by several spectroscopic methods [3.11.12]. Nevertheless, the exact structure is not known yet due to the fact that after the structural transformation the mechanically still very stable crystals have a mosaic type of architecture so that a detailed crystal structure investigation by $\mathrm{X}$-ray has not been possible up to now. The mosaic-type arrangement is a result of strong stress during the transformation because the structures of $\alpha-(\text { BEDT }- \text { TTF })_{2} I_{3}$ and $\beta-(B E D T-T T F)_{2} I_{3}$ are quite different from each other. Nevertheless, the unit cell data of $\beta-(\text { BEDT - TTF })_{2} I_{3}$ and $\alpha_{t}-(\text { BEDT -TTF })_{2} I_{3}$ and the arrangement of the molecules are the same. On the other hand the structure of $\alpha_{t}-(\text { BEDT }- \text { TTF })_{2} I_{3}$ cannot be exactly the same as in $\beta-$ (BEDT-TTF) ${ }_{2} I_{3}$ (or more correctly as in $\beta_{H^{-}}(B E D T-T T F)_{2} I_{3}$ ), since $\beta_{\mathrm{H}^{-}}$(BEDT - TTF $)_{2} \mathrm{I}_{3}$ has only a metastable superconducting state at $8 \mathrm{~K}$ while crystals of $\alpha_{t}-(\text { BEDT }- \text { TTF })_{2} I_{3}$ have a stable superconducting state at this temperature. The difference in the 
two structures exists probably only in the ordering of the terminal ethylene groups of the BEDT-TTF molecules. While in $\beta_{\mathrm{H}^{-}}(\mathrm{BEDT}-\mathrm{TTF})_{2} \mathrm{I}_{3}$ all the terminal ethylene groups have the so-called staggered form [13], in the crystals of $\alpha_{t}-$ (BEDTTTF) ${ }_{2} \mathrm{I}_{3}$ these groups might have the so-called eclipsed form or even more plausibly they might be ordered as in the $\alpha$-phase, where both forms of ordering exist in neighbouring stacks [14]. That means that very small changes in the structure such as a different ordering of the terminal ethylene groups might be responsible for the fact that the material has a stable or metastable superconducting state.

From the measurements of the upper critical fields of crystals of $\alpha_{t}-(B E D T-T T F)_{2} I_{3}$ it can clearly be seen that for the region $T / T_{c}>0.65\left(T_{c}=8 \mathrm{~K}\right)$ the crystals behave as isotropic three-dimensional superconductors, since for the magnetic field perpendicular and parallel to the $c^{*}$-axes the upper critical fields are identical. In the region $0.45<\mathrm{T} / \mathrm{T}_{c}<0.65$ an anisotropic three-dimensional behaviour is found while for $T / T_{c}<0.45$ the material can be described in the picture of typical two-dimensional layered superconductors [10]. By applying the anisotropic effective mass model of the Ginzburg-Landau (GL) theory the coherence lengthis $\xi_{11}(0) \simeq 120 \AA$ and $\xi_{1}(0) \simeq 20 \AA$ and the London penetration depths $\lambda_{11}(0)=4300 \AA$ and $\lambda_{1}(0)=750 \AA$ were obtained. However, since the anisotropic GL theory is only valid sufficiently close to $T_{c}$. these values can give only the right order of magnitude. Nevertheless. the value of $\xi_{1} \simeq 20 \AA$ is comparable to the unit cell dimension in the $\mathrm{c}$-direction. In addition ${ }^{13} \mathrm{C}-\mathrm{NMR}$ investigations [12.15] indicate that $\xi_{1}$ might even be only between 6 and $10 \AA$ since it was shown that the spin density (and therefore to some degree the charge density) is mainly located on the middle part of the BEDT-TTF molecules. This means that the thickness of the superconducting layer is of the order of the length of the BEDT-TTF molecules, supporting the picture of the Josephson coupled two-dimensional layered superconductors as described by Klemm et al. [10].

As pointed out before, a close similarity exists between the organic superconductors and the high- $T_{c}$ copper oxides. In both materials short coherence lengths of the order of the size of the unit cell and large London penetration depths exist. These properties are due to the typical quasi two-dimensional electronic conditions and structural arrangement. Certainly, in the case of the organic superconductors minor changes and differences in the structure, for instance a different ordering of the terminal ethylene groups of the BEDT-TTF molecules or a different sym- 
metry of the anions, might strongly influence the superconducting transition temperature. On the other hand such small variations in the structure of the organic materials open up the chance to obtain a larger number of materials with different transition temperatures into a superconducting state and variation of these structural parameters might be a way of obtaining higher transition temperatures in the case of the organic materials as well.

Nevertheless, a major difference exists between samples of the high $T_{c}$ copper oxide materials and the organic superconductors. The former are usually polycrystalline or granular and single crystals are difficult to obtain; in the latter case all physical investigations were done on single crystals. The electrochemical preparation of these organic metals results in single crystals suitable for physical investigations. But since the organic superconductors have very short coherence lengths as well, it should be possible to prepare pressed samples from polycrystalline organic metals - as in the case of the "ceramics" - which should exhibit bulk superconductivity.

\section{Properties of Polycrystalline Pressed Pellets of Organic Metals}

Polycrystalline pressed samples of size $4 \times 1 \times 0.5 \mathrm{~mm}^{3}$ were prepared from grained single crystals of the organic metals $\alpha_{t^{-}}$ and $\beta-(B E D T-T T F)_{2} I_{3}$ by applying a pressure of $(3-10) \cdot 10^{3}$ $\mathrm{kg} / \mathrm{cm}^{2}$ to the powder. The samples thus prepared are mechanically very stable and the temperature dependence of the resistivity can be measured easily by the usual four point method.

In the case of pressed samples prepared directly from $\alpha_{t}-($ BEDTTTF $)_{2} I_{3}$ no bulk superconductivity could be found. On the other hand in these samples a metal-like behaviour was observed over the whole temperature range between 300 and $1.3 \mathrm{~K}$. This fact is at least worth noticing since usually in polycrystalline pressed pellets of organic conductors only a semiconducting behaviour is found (this is specially valid for quasi one-dimensional materials).

Since from the single crystal investigations it was known that small changes in the structure might suppress the superconductivity, it was assumed that during the preparation of the polycrystalline pellets of $\alpha_{t}-(B E D T-T T F)_{2} I_{3}$ under pressure phase transitions might occur. Therefore, newly prepared pellets of $\alpha_{t}-(\text { BEDT - TTF })_{2} I_{3}$ were annealed for 3 days at $75^{\circ} \mathrm{C}$ before the samples were provided with contacts and measured. After this procedure a superconducting transition could be observed, but compared with the crystal of $\alpha_{t}-(B E D T-T T F)_{2} I_{3}$ the supercon- 
ducting transition in the polycrystalline sample is much broader [16]. Zero resistivity is found at $2.2 \mathrm{~K}$ and the middle of the transition appears at about $5.5 \mathrm{~K}$, while in the case of the crystals of $\alpha_{t}-(\text { BEDT - TTF })_{2} I_{3}$, zero resistivity appears already at $6 \mathrm{~K}$ and the middle of the transition occurs at $8 \mathrm{~K}$ [3].

The most surprising observation was made by measuring the temperature dependence of the resistivity of polycrystalline pressed samples of $\beta-(B E D T-T T F)_{2} I_{3}$ (in the following called $\beta_{p}-(B E D T-$ TTF) ${ }_{2} I_{3}$ ) [17]. Without annealing the samples showed an onset to superconductivity at $9 \mathrm{~K}$, zero resistivity at $3.2 \mathrm{~K}$ and the middle of the resistive transition at $7.5 \mathrm{~K}$ [17]. This observation is surprising because single crystals of $\beta-(B E D T-T T F)_{2} I_{3}$ show a rather sharp stable superconducting transition but only at $1.4 \mathrm{~K}$ [18] and a metastable superconducting state at $8 \mathrm{~K}[19,20]$. Here in the polycrystalline pressed pellets of $\beta_{\mathrm{p}}-(\mathrm{BEDT}-\mathrm{TTF})_{2} \mathrm{I}_{3}$ the superconducting state at $7.5 \mathrm{~K}$ is stable and a bulk effect of the pellets [17].

The resistivity characteristics of the polycrystalline pressed pellets of $\beta_{\mathrm{p}}-(\mathrm{BEDT}-\mathrm{TTF})_{2} \mathrm{l}_{3}$ [17] indicate that the detailed structure of $\beta_{\mathrm{p}}-(\mathrm{BEDT}-\mathrm{TTF})_{2} \mathrm{I}_{3}$ is different to the one of the polycrystalline pressed $\alpha_{t}-(B E D T-T T F)_{2} I_{3}$ and different to the single crystals of $\beta-(B E D T-T T F)_{2} I_{3}$, even if the unit cell data are identical and the molecular arrangement in the materials might be very similar. A possible difference might lay again in the ordering of the terminal ethylene groups of the BEDT-TTF molecules (we assume at the moment that all ethylene groups are ordered in the eclipsed form ).

\section{Acknowledgement}

I would like to thank all my colleages and co-workers (see references) for very effective cooperation during all the investigations in recent years. My special thanks go to Professor H. J. Keller for many discussions and efficient teamwork.

\section{References}

1. D. Jérome, A. Mazaud. M. Ribault and K. Bechgaard, J. Phys. Lett. $\underline{4}$, L. 95 (1980).

2. H. Urayama, H. Yomochi. G. Saito, K. Nozava, T. Sugano, M. Kinoshito, S. Saito. K. Oshima, A. Kawamoto and J. Tanaki, Chem. Lett 1988, 5 (1988). 
3. D. Schweitzer, P. Bele, H. Brunner, E. Gogu. U Haeberlen, 1. Hennig, T. Klutz, R. Swietlik and H. J. Keller, Z. Phys. B Condensed Matter 67. 489 (1987).

4. K. Murata, M. Tokumoto, H. Anzai, H. Bando, G. Saito, K. Jajimura and T. Ishiguro, J. Phys. Soc. Japan 54. 1236 (1985).

5. V.N. Laukhin. E.E. Kosty'nchenko, Yu.Y. Sushko. I. F. Shegolev and E. B. Yagubskii, Soviet Physics JETP Lett. 41. 81 (1985).

6. S. Gärtner, E. Gogu, I. Heinen, H. J. Kefler, T. Klutz and D. Schweitzer, Solid State Comm. 65, 1531 (1988).

7. S. Klotz, J. S. Schilling. S. Gärtner and D. Schweitzer, Solid State Comm. 67, 1981 (1988).

8. K. Oshima, H. Urayama, H. Yanochi and G. Saito. J. Phys. Soc. Japan 57, 730 (1988).

9. E. Gogu. Ph. D. Thesis, Universität Heidelberg 1989.

10. R. A. Klemm, A. Luther and R. M. Beasley. Phys. Rev. B 12 . 877 (1975).

11. G. O. Baram, L. I. Buravov, L. C. Degtariev, M. E. Kozlov, V.N. Laukhin. E. E. Laukhina, V. B. Drischenko. K. L. Pokhodnia. M. K. Scheinkmann. R. P. Shibaeva and E. B. Yagubskii, Sov. Phy's. JETP Lett. $\underline{4 t}, 2913$ (1986).

12. I. Hennig, U Haeberlen, I. Heinen, D. Schweitzer and H.J. Keller; Physica C 153-155, 493 (1988).

13. A. J. Schultz, H. H. Wang, J. M. Williams and A. Filhol, J. Am. Chem. Soc. 108, 7853 (1986).

14. K. Bender, I. Hennig, D. Schweitzer, K. Dietz, H. Endres and H.J. Keller, Mol. Cryst. Liqu. Cryst. 108, 359 (1984).

15. D. Schweitzer, K. Polychroniadis, T. Klutz, H. J. Keller, I. Hennig, I. Heinen, U. Haeberlen, E. Gogu and S. Gärtner, Synth. Metals 27, A 465 (1988).

16. D. Schweitzer, S. Gärtner, H. Grimm, E. Gogu and H. J. Keller, Solid State Comm. 69, 843 (1989).

17. D. Schweitzer, E. Gogu, H. Grimm, S. Kahlich and H. J. Keller, Angew. Chem. Adv. Mater. 101, 977 (1989).

18. E. B. Yagubskii, I. F. Shegolev, V.N . Laukhin, P. A. Kononovich, M. V. Kartsovnik. A. V. Zwarykina, L. I. Biwavov, Sov. Phys. JETP Lett. 39, 12 (1984).

19. F. Creuzet. G. Creuzet, D. Jèrome, D. Schweitzer and H. J. Keller, J. Physique Lett. 46, L -1079 (1985).

20 F. Creuzet. D. Jèrome, D. Schweitzer and H. J. Keller, Europhys. Lett. 1,461 (1986). 